\title{
Zonal jets in rotating shallow water turbulence
}

\author{
G. Di Nitto, S. Espa, A. Cenedese \\ DICEA, “Sapienza” University of Rome, Rome, Italy
}

Email address:

gabriella.dinitto@uniroma1.it (G. Di Nitto), stefania.espa@uniroma1.it (S. Espa), antonio.cenedese@uniroma1.it (A. Cenedese)

\section{To cite this article:}

G. Di Nitto, S. Espa, A. Cenedese. Zonal Jets in Rotating Shallow Water Turbulence, Earth Science. Vol. 2, No. 2, 2013 , pp. 23-30. doi: $10.11648 /$ j.earth.20130202.11

\begin{abstract}
During the last three decades, the appearance of multiple zonal jets in planetary atmospheres and in the Earth's oceans has widely studied. Evidences of this phenomenon were recovered in numerical simulations [1], laboratory experiments [2-4] and in field measurements of giant planets' atmosphere [5]. Recent studies have revealed the presence of zonation also in the Earth's oceans; in fact, zonal jets were recovered in the outputs of Oceanic General Circulation Models-GCMs [6] and from satellite altimetry observations [7]. In previous works [3-4], we have investigated the impact of several experimental parameters on jets organization both in decaying and forced regimes. This work shows new results in the context of continuously forced flows obtained performing experiments in a bigger domain. The experimental set-up consists of a rotating tank where the initial distribution of vorticity is generated via the Lorentz force in an electromagnetic cell and the latitudinal variation of the Coriolis parameter is simulated by the parabolic profile assumed by the free surface of the rotating fluid. The velocity fields were measured using an image analysis technique. The flow is characterized in terms of zonal and radial flow pattern, flow variability and jet scales.
\end{abstract}

Keywords: Zonal Jets, B-Plane Turbulence, Laboratory Experiments

\section{Introduction}

The presence of alternating zonal jets is a very common feature of the planetary atmospheres and the terrestrial oceans. In freely decaying conditions, the spontaneous appearance of zonal jets has been highlighted, firstly, by Rhines [8]. He showed that the latitudinal variation of the Coriolis parameter, induces an anisotropization of the inverse energy cascade and the energy is transferred towards slowest modes accumulating at a wavenumber, called Rhines scale $\mathrm{k}_{\mathrm{Rh}}$. In correspondence of this scale, the energy is channeled into zonal jets and Rossby waves are emitted. Furthermore, Rhines supposed the arrest of the inverse energy cascade at $\mathrm{k}_{\mathrm{Rh}}$.

Sukoriansky et al. [9] have recently revisited this concept considering a small-scale forced, barotropic, dissipative, two-dimensional flow, subjected to the $\beta$-effect, in which an inverse energy cascade develops. They demonstrated that the $\beta$-effect causes only the anisotropization of the inverse cascade rather than its halting. In consequence, the flow may attain several steady-state regimes which can be classified in terms of the characteristic wavenumbers associated with the forcing, $\mathrm{k}_{\mathrm{f}}$, the small-scale dissipation, $\mathrm{k}_{\mathrm{d}}$, the $\beta$-effect, $\mathrm{k}_{\beta}$, and the large-scale drag, $\mathrm{k}_{\mathrm{fr}}$. Among these regimes, a universal behavior has been recovered in the so-called zonostrophic regime that can be considered as a subset of the geostrophic turbulence. Its main characteristics are a strongly anisotropic kinetic energy spectrum and a slowly changing system of alternating zonal jets spanning the entire flow domain. A zonally banded flow pattern can be found also in intermediate regimes.

In this paper, we investigated experimentally the appearance of zonal jets in a continuously forced, turbulent rotating shallow fluid. In particular, we focused on how the variation of external parameters, like the fluid thickness and rotation rate, affects the formation/evolution of zonal jets.

\section{The $\beta$-plane turbulence}

The theoretical work of Rhines [8] represents a fundamental reference for the study of rotating $2 \mathrm{D}$ turbulence. $\mathrm{He}$ showed how the presence of a strong rotation, and consequently the presence of the $\beta$-effect, modifies significantly the dynamics of 2D turbulence, either through the generation of Rossby waves or the stabilization of the zonal flow. In addition, Rhines was the first who recognized the role of both these aspects on the anisotropization - i.e. the preferential energy transfer towards zonal modes - of unforced barotropic rotating flows. One of the most important results 
of his work is the identification of the dominant scale, denoted successively as Rhines scale $\left(\mathrm{k}_{\mathrm{Rh}}=\left(\beta / 2 \mathrm{U}_{\mathrm{rms}}\right)^{1 / 2}\right)$, at which the root mean square of the velocity $\left(\mathrm{U}_{\mathrm{rms}}\right)$ is equal to the phase speed of Rossby waves. Rhines supposed an energy spectrum divided into two ranges, $\mathrm{k}>\mathrm{k}_{\mathrm{Rh}}$ where turbulent effects dominate and $\mathrm{k}<\mathrm{k}_{\mathrm{Rh}}$ characterized by wave-like phenomena. The Rhines scale was also associated with the flow organization into alternating zonal jets (zonation) whose width scales with $\mathrm{k}_{\mathrm{Rh}}{ }^{-1}$.

Based on the barotropic vorticity equation, 2D turbulence with a $\beta$-effect has been numerically studied in decaying and forced regimes, both on a rotating sphere and on a $\beta$-plane. Simulations in decaying regime are useful for studying inviscid dynamics but they are perhaps too idealized for real world applications [10-12]. In the planetary atmospheres, in fact, energy is continuously supplied so forced turbulent models are more suitable for studying the atmosphere of an Earth-like planet [1, 12-15].

As far as simulations in decaying regime are concerned, Yoden and Yamada [10] studied the problem on a rotating sphere. They showed a dependence of the temporal evolution of the flow field on the rotation rate. In particular, a non-rotating case $(\Omega=0)$ they observed isolated coherent vortices; on the contrary, as $\Omega$ is increased an anti-cyclonic circumpolar vortex formed.

Cho and Polvani [11] described a freely decaying shallow-water system on a rotating sphere. In a non-divergent flow, that corresponds to the limit $\mathrm{L}_{\mathrm{D}} \rightarrow \infty$ where $\mathrm{L}_{\mathrm{D}}$ is the Rossby deformation radius $\left(\mathrm{L}_{\mathrm{D}}=(\mathrm{gH})^{0.5} / \Omega\right.$, with $\mathrm{H}$ the fluid thickness), they observed the formation of some zonal jets, at low rotation rate. These jets are not steady, since vertical structures are not well confined in the meridional direction. In the presence of sufficiently strong rotation, the non-divergent long-time behavior yields a field dominated by anticyclonic circumpolar vortices. Considering a rotating fluid bounded by a free surface, i.e. a finite Rossby deformation radius, they observed the evolution of the flow toward a robust banded configuration, in which the number of bands increases with the rotation rate, as predicted by Rhines. As Cho and Polvani pointed out, the bands are not perfectly zonal; in fact, they spiral at high latitudes, unlike in the forced $\beta$-plane simulations.

Based on Rhines' predictions many attempts to generate persistent zonal jets have been made also in models of forced two-dimensional turbulence. It is important to underline that in this case the presence of a small scale forcing selects a new scale $\left(\mathrm{k}_{\beta}\right)$ as the transition between turbulence and waves [1].

In this context, studying barotropic planetary zonal jets, Williams [14] simulated multiband zonal jets that broadly resemble the zonal flow of the Jovian atmosphere in a 2D turbulent model with stochastic forcing at small scales. He used only a sector model (e.g. on one-sixteenth of the surface of a sphere) reporting results periodically repeated (Fig. $1)$.

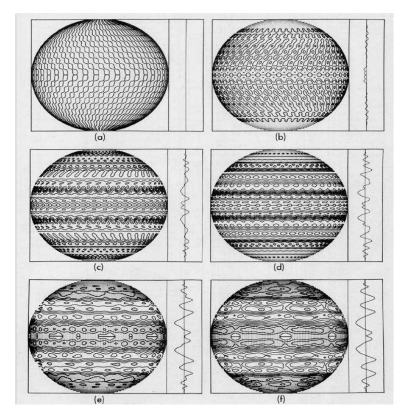

Figure 1. Plot of the stream function at different time instants. Zonally averaged zonal velocity profiles are plotted at right [14].

Vallis and Maltrud [1] examined turbulence-waves transition on the $\beta$-plane using a better numerical resolution than the one used in the original studies by Rhines. With different scaling theories, they found three relationships for the transition wavenumber (one of them recovers the Rhines expression). Considering the scaling based on the classical, double cascade of two-dimensional turbulence, they found the expression of $\mathrm{k}_{\beta}$ equating the Rossby wave period with the turbulent eddy turnover time in the regime of isotropic 2D turbulence $\left(\mathrm{k}_{\beta}=\left(\beta^{3} / \varepsilon\right)^{1 / 5}\right)$. In their numerical experiments, they also observed the development of zonal jets in remarkably stable position. They found that the jet scale decreases as the input energy is decreased (or $\beta$ is increased), and it is generally of the same order as the Rhines scale. In their banded configurations, Vallis and Maltrud observed a marked asymmetry, with the eastward jets narrower and faster than the westward jets, consistent with the stability criterion. In terms of energy, they observed a piling-up of the computed energy spectrum near $\mathrm{k}_{\beta}$.

The dynamics of forced two-dimensional turbulence in spherical geometry were examined by Huang and Robinson, Nozawa and Yoden, $[12,16]$. In their numerical simulations, they obtained very steady zonal jets in which the jet spacing decreases with the forcing amplitude, consistently with the phenomenology described by Vallis and Maltrud and by Panetta [1, 17].

Recent numerical studies, focused on small-scale forced, barotropic, dissipative, two-dimensional turbulence with a $\beta$-effect [5, 9, 18-21], have led new important insight into the understanding of the large-scale planetary circulations. It has been found that this type of flows may attain several steady-state regimes. In particular, the ratio between the transitional wave number and the Rhines wave number $\left(\mathrm{R}_{\beta}=\mathrm{k}_{\beta} / \mathrm{k}_{\mathrm{Rh}}\right.$, coined as zonostrophy index), selects the nature of the flow regime. Four possible regimes have been identified in the parameter space $\left(\mathrm{k}_{\beta}, \mathrm{k}_{\mathrm{Rh}}\right)$. Two of them are universal regimes: the friction-dominated regime $\left(\mathrm{R}_{\beta}<1.5\right)$ and the zonostrophic regime $\left(R_{\beta}>2.5\right)$. The latter one is characterized by a large enough inertial range, a sufficiently wide scale separation between the forcing scale and scales influenced by the $\beta$-effect, and a large enough frictional wave number to avoid the large-scale energy condensation $[18,19]$. The zonostrophic regime is distinguished by a strongly anisotropic kinetic energy spectrum whose zonal 
mode alone may contain more energy than all other modes combined [18, 21, 23]. The most distinguished visual feature of zonostrophic turbulence is the formation of a nearly stable system of eastward/westward zonal jets in the flow domain.

As far as experimental studies are concerned, jet flows on the $\beta$-plane have been pointed out in several experiments based on flow measurements by image analysis. Whitehead [24] was the first who studied experimentally the $\beta$-plane turbulence in 1975 . He simulated the polar $\beta$-plane by rotating a cylindrical container of water with a free surface such that the depth of the layer varies parabolically with the radius. In these pioneering experiments, jet flows were obtained with a variety of mechanisms. A locally and vertically oscillating disc produced a narrow eastward jet at the latitude of the forcing and a broad westward flow outside of this region. A flow qualitatively very similar to the one generated by the plunger, was obtained with air bubbles rising from a pipe with 20 holes located $60 \mathrm{~cm}$ from the center of the tank.

The classical method based on the differential heating of a rotating annulus was used extensively in order to reproduce zonal flows [25]. With this type of forcing, a cylindrical insert is typically placed in the center of an annulus. The inner walls are then cooled or the outer walls heated, or a combination of both. Since the cold water is denser (and thus heavier) than the warm water, the cold water sinks at the inner wall while warm water rises at the outer wall. This results in a radial flow that is directed towards the center of the container at the surface of the layer and towards the outer walls at the bottom of the container. These radial motions are then converted into zonal flows by the presence of the Coriolis force. Using this type of forcing, Bastin and Read [26] were able to observe the formation of zonal jets as well as some stable eddies over a range of rotation rates. Wordsworth et al. [27] showed the formation of multiple zonal jets at mid-depth when sloping top and bottom boundaries were used in the rotating annulus. They also showed that the eddies exchange energy with the zonal modes directly and non-locally in spectral space.

Aubert et al. [28] studied experimentally and numerically a rotating fluid in which a zonal flow emerged from small-scale eddies. They used an array of sinks and sources located along a ring in the middle of a rotating annulus with a sloping bottom. This type of forcing generated vortex filaments of the same width as the holes that merged and mixed potential vorticity (PV) in the inner and outer regions of the annulus. Snapshots of the measured potential vorticity are shown in Fig. 2: strong cyclones (red) and anticyclones (blue) formed. A retrograde drifting Rossby wave prevented mixing between the two regions, visible particularly in numerical simulations (Fig 2-b). The strongly forced flow was dominated by vortices so no zonal pattern was apparent. However, the authors observed a zonal flow pattern performing time averages. In particular, they found three areas for azimuthal flow: a prograde zonal circulation in correspondence of the PV gradient region, a retrograde zonal circulation in the regions of approximately constant PV.

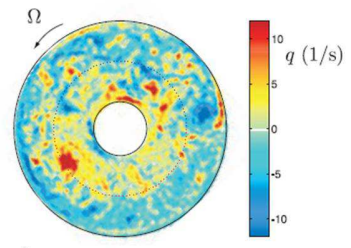

a)

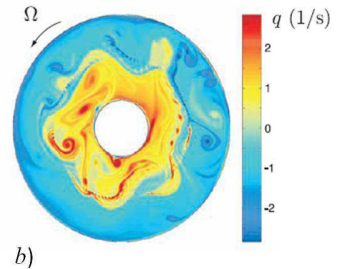

b)
Figure 2. The potential vorticity fields obtained from: a) experiment; b) numerical simulation [28].

Electromagnetic (EM) forcing of conducting fluids is a non-intrusive technique, used to study a large variety of flows. In particular, EM forcing has been used in shallow layers of electrolytes to study quasi-two-dimensional turbulence [29-31], shallow vortices [32], the principles of stretching and folding in quasi-two-dimensional flows [33]. In the first reported experiments of this kind, it was found that the induced fluid velocity was proportional to the magnitude of the EM forcing [34]. EM forcing was used also to study $\beta$-plane turbulence in decaying $[2,3,35,36]$ and forced regimes [4]. In this paper we will show results from experiments performed in a rotating electromagnetic cell.

In Afanasyev and Wells [2] it was shown that a zonal flow, in the form of a strong prograde jet and relatively weak retrograde circulation, emerged from the initially turbulent flow of $\beta$-plane turbulence. In the experiments with a relatively high value of the $\beta$-parameter, the characteristic behavior of the flow, in a decaying regime, was the formation of a polar vortex strongly perturbed by (stationary) Rossby waves (Fig. 3-a). In the experiments with a low value of the $\beta$-parameter, the potential vorticity in the center of the container was found to be mixed to a significant degree and the evolution of the flow was found to be similar to that of non-rotating quasi-two-dimensional turbulence (Fig. 3-b). Similar results have been found in Espa et al. [3, 35] and in [36] in which the authors studied also the asymmetry between cyclonic/anticyclonic vortices. EM forcing in a forced regime was used in Espa et al. [4] in order to study small-scale turbulence on the $\beta$-plane in a more realistic configuration, since in nature the energy is continuously supplied. The present work is on the wake of this first forced study.

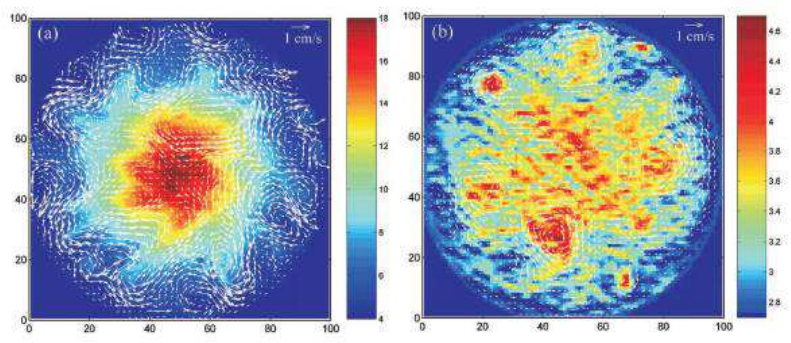

Figure 3. Vorticity (color) and velocity (arrows) fields measured in two experiments with high and low beta at $t=4 \mathrm{~s}$ after the forcing was stopped [2]. 
Read et al. [37] reproduced multiple zonal jets on the $\beta$-plane in a convectively driven laboratory flow at the large-scale Coriolis facility, in Grenoble. They produced a turbulent flow gently and continuously spraying dense salty water onto the surface of a cylindrical tank $(13 \mathrm{~m}$ in diameter). The $\beta$-effect is obtained by means of a conically sloping bottom and the small deformations of the free surface due to the rotation. After several hours, they observed a zonally banded large-scale flow pattern (Fig. 4) characterized by spectral anisotropization. The flow has been identified as marginally zonostrophic $\left(\mathrm{R}_{\beta}=0.5-2\right)$. From an experimental point of view, the Grenoble experiment is the most representative attempt to reproduce in the laboratory the best conditions for the establishing of the zonostrophic regime.

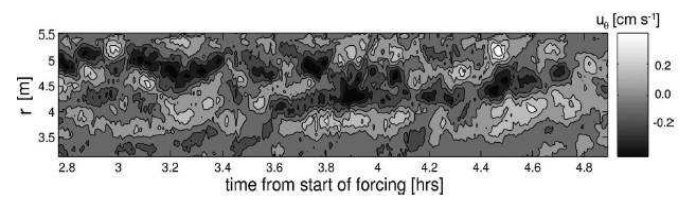

Figure 4. Azimuth-time contour plot of mean azimuthal flow at mid-height in the Coriolis tank, for strong $\beta$-case, over a period of approximately $2 h$. Shaded contours are of azimuthal velocity $u_{\theta}\left(\mathrm{cms}^{-1}\right)$ [37].

Recently, different mechanisms of forcing were used in order to show a mechanism whereby the jets result during the development of the so-called $\beta$-plumes. A $\beta$-plume consists of an eastward and westward jet at its Northern and Southern flanks [38]. In Afanasyev et al. [38] the authors analyzed different configurations of the flow, including the flows induced by single localized sources of buoyancy located at the "mid-latitude" of the tank, flows due to coastal current flowing along the radial barrier representing the eastern boundary and flows generated by the linear source of buoyancy. In all the experiments jets occurred as a result of initially linear development of $\beta$-plumes. The distance between jets seems to be determined by the size of the original perturbation that generates the $\beta$-plume. The formation of multiple jets was observed also in experiments with a spatially localized heater (Fig. 5) [39].

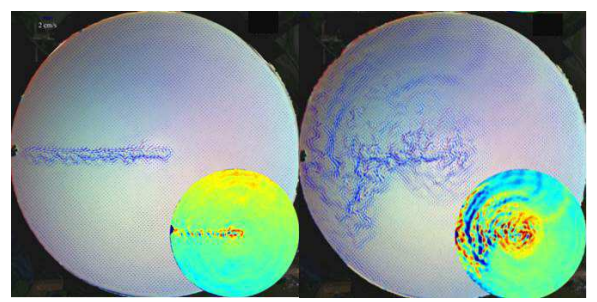

(a)

(b)

Figure 5. Sequence of velocity fields in an experiment measured at $t=39 \mathrm{~s}$ (a) and $1200 \mathrm{~s}$ (b). The velocity vectors are superposed on the altimetry images of the fluid surface. The inserts show the color maps of azimuthal velocity varying from -0.8 (blue) to $0.8 \mathrm{~cm} / \mathrm{s}$ (red) such that yellow-red bands indicate eastward jets while blue bands indicate westward jets [39].

\section{Experimental Set-Up}

The experiments have been performed in a square tank, whose internal dimensions are $69 \mathrm{~cm} \times 68 \mathrm{~cm} \times 15 \mathrm{~cm}$, placed on a rotating table ( $1 \mathrm{~m}$ in diameter). In order to simulate flows in the Northern hemisphere, a rotation in a counterclockwise direction was imposed. The parabolic free surface assumed by the fluid under rotation is used to model in laboratory the variation of the Coriolis parameter with latitude ( $\mathrm{f}(\mathrm{y})$, where $\mathrm{y}$ is the meridional coordinate), near the poles. It can be shown that, due to the PV conservation, there is an exact dynamical equivalence between the variation of the Coriolis parameter with latitude, and the variation of height in the presence of constant $f(f=2 \Omega$ in the laboratory) [40]. The dynamics associated with the Coriolis parameter in the polar region is captured by a quadratic variation of it in $r$, the radial distance from the pole, assuming the pole as the reference point (this is the so-called $\gamma$-plane or polar- $\beta$-plane approximation) [40]:

$$
\mathrm{f}(\mathrm{y})=\mathrm{f}_{0}+\gamma \mathrm{r}^{2}
$$

where $f_{0}=2 \Omega \sin \varphi_{0}, \varphi_{0}$ is the central latitude of the domain considered, $\gamma=\Omega / \mathrm{R}^{2}, \Omega$ and $\mathrm{R}$ are the rotation and the radius of the Earth.

In a rotating free-surface fluid, the fluid depth is defined as $\mathrm{H}(\mathrm{r})=\mathrm{H}_{0}\left(1+\gamma\left(\mathrm{r}^{2}-\left(\mathrm{L}^{2}+\mathrm{W}^{2} / 12\right)\right)\right)$ where $\mathrm{H}_{0}$ is the mean depth of the fluid, $\gamma=\mathrm{f}^{2} /\left(8 \mathrm{gH}_{0}\right)$ is the analogue of the $\gamma$-parameter defined in $(1), \mathrm{f}_{0}=2 \Omega, \mathrm{L}$ and $\mathrm{W}$ are the dimensions of the tank [38]. The point of maximum depression of the fluid surface represents the pole, while the periphery of the domain corresponds to the lower latitudes. Using this experimental configuration, the resulting flow is then aimed to simulate the dynamics in correspondence to the polar region of a rotating sphere (indicatively $45^{\circ} \leq \varphi \leq$ $90^{\circ}$ ).

In this frame of reference, for intermediate latitudes, the expression of the $\beta$-term (the northward gradient of the Coriolis parameter in the linear approximation) is:

$$
\beta=2 \mathrm{sr}_{\mathrm{m}} \mathrm{f}_{0} / \mathrm{H}\left(\mathrm{r}_{\mathrm{m}}\right)
$$

where $r_{m}=R / 2$ is a mid-latitude reference point being $R$ the maximum radial distance, $\mathrm{s}=\Omega^{2} / 2 \mathrm{~g}[2,3]$.

In our experiments, a turbulent flow was produced non-intrusively by means of an electromagnetic forcing. The tank was filled with an electrolyte solution $\left(\mathrm{C}_{\mathrm{NaCl}}=\right.$ $80 \mathrm{~g} / \mathrm{l})$ to a depth of $3 \mathrm{~cm}$ or $4 \mathrm{~cm}$. An array of axially magnetized permanent (neodymium) magnets was placed underneath the fluid layer with a spacing of $3 \mathrm{~cm}$ and alternating polarity. We used a combination of circular $(\varnothing=1.1 \mathrm{~cm}$, $\mathrm{H}=0.5 \mathrm{~cm})$ and rectangular magnets $\left(\mathrm{L}_{\mathrm{x}} \times \mathrm{L}_{\mathrm{y}} \times \mathrm{H}=2 \times 1 \times\right.$ $0.5 \mathrm{~cm}^{3}$ ), whose magnetic field strengths were, respectively, of approximately $1191.5 \mathrm{G}$ and $1232.3 \mathrm{G}$ at the center of the magnet surface. Since the depth of the rotating fluid varied parabolically with the radius, in the inner part of the tank we placed the circular magnets, while in the external part the more intense rectangular ones. When the fluid was spun up to the solid-body rotation at a constant rate $\Omega$, a constant voltage was applied to two electrodes placed inside the tank, 
on opposite sidewalls. The interaction between the horizontal current and the vertical magnetic field generated a Lorentz force perpendicular to both fields that set the fluid in motion. The initial vorticity distribution was characterized by the formation of opposite signed eddies, positive or negative according to the phase of the resulting Lorentz force, and whose initial horizontal length scales were related to the dimensions of the magnets and the distance between them.

To investigate the turbulence by means of a non-intrusive image analysis technique the fluid surface was seeded with styrene particles with a mean diameter of about $50 \mu \mathrm{m}$. The tank was covered with a transparent lid to prevent interaction with air. The free surface was lit with two lateral lamps to have a high contrast between the white particles and the black bottom. A video camera co-rotating with the system, perpendicular to the tank and with the optical axis parallel to the rotation axis, recorded the experiments with a frame rate of 20 frame/s and a resolution of $1023 \times 1240$ pixel. The image analysis technique, called Feature Tracking, allowed reconstructing the velocity field evolution in a Lagrangian framework. Then, the velocity fields were interpolated onto a regular Eulerian $128 \times 128$ grid. Technical details on the measuring technique are described in [41]. Several experiments were performed by changing external parameters in order to analyze their impact on the characteristics of the flow. For a fixed initial depth $\left(\mathrm{H}_{0}=3 \mathrm{~cm}-4 \mathrm{~cm}\right)$ and an input current of $8 \mathrm{~A}$, we varied the tank rotation rate in the range $2.0 \mathrm{~s}^{-1} \leq \Omega \leq 3.1 \mathrm{~s}^{-1}$.

The forcing is applied continuously for all the duration of the experiments $(6 \mathrm{~min})$. The control non-dimensional parameters of the experiments are: the aspect ratio $(\delta=\mathrm{H} / \mathrm{L})$ that falls in the range 0.04-0.06; the Ekman number (Ek = $v / \Omega \mathrm{H}^{2}$ where $v$ is the kinematic viscosity of the fluid) that is of the order of $10^{-4}$; the Reynolds number $(\mathrm{Re}=\mathrm{UL} / \mathrm{v}$, where $U$ is the mean absolute value of the total velocity and $\mathrm{L}$ is the length of the tank) of the order of $10^{3}$ and the Rossby number $(\mathrm{Ro}=\mathrm{U} /(2 \Omega \mathrm{L}))$ of the order of $10^{-3}$.

\section{Results}

\subsection{Radial And Zonal Flow Pattern}

The geometry of our system suggests using a polar coordinate system with the pole in correspondence of the center of the tank. In this frame of reference, a distance from the pole and an angle from a fixed direction (center of the tank-East) determine each point on the plane. The distance from the pole is called the radial coordinate or radius $(r)$; the angle is the angular coordinate or azimuth $(\theta)$. The eulerian velocity fields are interpolated onto a polar grid created using 360 radii and 60 circles. For each point of the grid, the velocity is decomposed into the radial (parallel to the radius) and azimuthal (tangential to the curve) components. The azimuthal component of the velocity is referred to as the zonal component. In a rotating fluid points with the same height are aligned along circles that correspond to planetary parallels (points with the same latitude in a rotating sphere). Thus, the azimuthal direction represents the zonal direction.

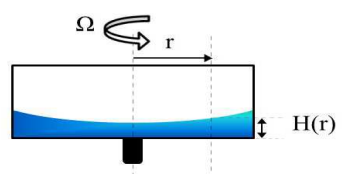

Figure 6. Sketch of the experimental set-up.

Fig. 7 shows a map of the zonal velocity on the polar grid for an experiment with a fast table rotation. The colors represent the zonal velocity; the arrows the total velocity field. It is possible to observe structures elongated in the azimuthal direction with opposite velocity along the radial direction.

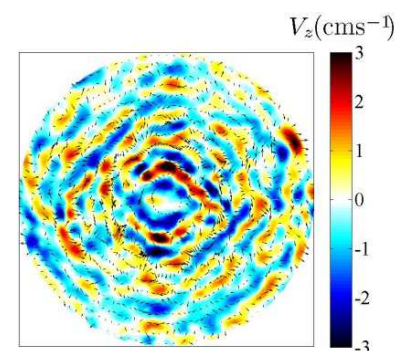

Figure 7. Zonal velocity map (colors) of a fast rotating experiment averaged over 1s The arrows represent the total velocity field.

In Fig 8 the instantaneous radial-azimuthal patterns of the zonal, $V_{z}$, (top panel) and the radial velocity, $V_{r}$, (bottom panel) are shown for the same experiment. The azimuthal velocity bands are homogeneous along azimuthal intervals. In the remainder of the paper, the interval $\theta=\left[0-180^{\circ}\right]$ is considered for zonal averaging. In all the experiments, we observed similar zonal patterns with some differences in terms of jet spacing.

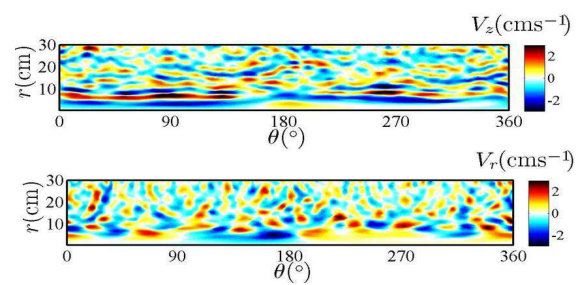

Figure 8. Instantaneous radial-azimuthal map of the zonal velocity (top panel); Instantaneous radial-azimuthal map of the radial velocity (bottom panel).

\subsection{Mean and Instantaneous Zonal Velocity Profiles}

An insight into the degree of anisotropy of the flow is gained by performing zonal averaging, that is averaging over $\theta$. In Fig. 9 the instantaneous (top panel) and time-mean (bottom panel) radial profiles of the mean azimuthal velocity for three experiments with different rotation rate are shown. The profiles are plotted, from left to right, with increasing values of $\beta$. The instantaneous profiles show the presence of alternating zonal bands also in the 
lower $\beta$ case (Fig. 9-a). The inner part of the tank is characterized by more intense zonal structures due to the stronger forcing intensity. It is possible to compare, qualitatively, the radial scale of the zonal structures. As expected, broader jets are recovered in the lower $\beta$ case. The bottom row of Fig. 9 shows the radial profiles of the mean zonal velocity, averaged over approximately 5 minutes of the late phase of the experiments. We performed time averages since we checked the establishing of a steady state with a statistical tool, the so-called reverse arrangement test [42].
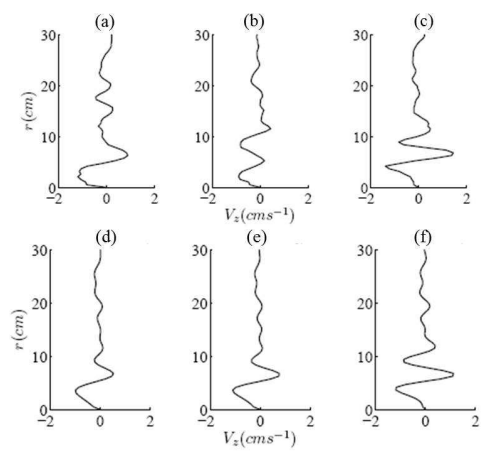

Figure 9. Instantaneous profiles of the mean zonal velocity (top panel); Time-mean profiles of the mean zonal velocity (bottom panel). Increasing values of $\beta$ from left to right (a-d) $\beta=0.14 \mathrm{~cm}^{-1} \mathrm{~s}^{-1}$; b-e) $\left.\beta=0.22 \mathrm{~cm}^{-1} \mathrm{~s}^{-1} ; c-f\right)$ $\beta=0.38 \mathrm{~cm}^{-1} \mathrm{~s}^{-1}$ ).

After time averaging, some zonal jet-like structures survive, especially in the inner part of the tank. We evaluated the root mean square of the zonal velocity; it ranges between $0.48 \mathrm{cms}^{-1}$ and $0.80 \mathrm{cms}^{-1}$.

\subsection{Zonal Energy and Jet Scale}

According to Huang and Robinson [12], the time-mean total energy can be decomposed by $\mathrm{E}=\mathrm{E}_{\mathrm{SZ}}+\mathrm{E}_{\mathrm{TZ}}+\mathrm{E}_{\mathrm{SE}}+$ $\mathrm{E}_{\mathrm{TE}}$, where the terms on the right-hand size of the equation represent the time mean of the stationary zonal energy, the transient zonal energy, the stationary eddy energy and the transient eddy energy, respectively. The term "eddy" means deviation from the zonal mean. The ratio of the zonal to the total energy does not vary greatly from case to case, with a typical value of around 0.5 ; this means a rough equipartition between the eddy and zonal energy. The scale of the zonal jets can be evaluated by the square root of the ratio between the zonal enstrophy and the zonal energy [12]. Generally, it decreases with decreasing energy, or increasing $\beta$, consistent with the phenomenology described by Vallis and Maltrud [1] and by Panetta [17]. In our experiments, we estimated $1.36 \mathrm{~cm}^{-1} \leq \mathrm{k}_{\mathrm{j}} \leq 1.66 \mathrm{~cm}^{-1}$, with increasing values of the jet scale for increasing $\beta$, as expected. Thus, the number of jets increases with increasing $\beta$.

\section{Conclusions}

In the last 30-40 years, the emergence of zonal jets in the planetary atmospheres and in the Earth's oceans and the geophysical implications due to their presence have been intensely studied, especially from a numerical point of view. In this context, we investigated the appearance of zonal jets in forced rotating shallow water turbulence in a laboratory setting. An experimental approach can lead new insight into the dynamics of this phenomenon due to the advantages related to the use of a controlled environment and the repeatability of the phenomena. The relevance of our work is due to the small number of laboratory studies aimed at the investigation of the zonal jets.

In this paper, we presented results from several experiments performed in a rotating electromagnetic cell. In order to investigate the effect of the $\beta$-strength on the flow evolution we varied different control parameters like the tank rotation rate and the fluid thickness. As a matter of fact, an increase of the background rotation (or a decrease of the fluid depth) leads to a stronger $\beta$-effect. It has been observed that as the $\beta$-effect increases, the flow tends to evolve in the zonal direction, i.e. the azimuthal direction. We were able to show the formation of alternating bands characterized by positive/negative mean zonal velocities. The flow shows a clear zonally banded structure for higher values of $\beta$. We evaluated the radial length scale of the jets as a function of the $\beta$-term. As expected, we found larger radial widths in cases of weak $\beta$-effect.

We are actually performing further analyses in order to better characterize the flow evolution. New insights will be led by the spectral analysis and the investigation of the eddy-mean flow interactions. We are evaluating the dependence of the zonal structures on other experimental parameters such as the forcing amplitude. Moreover, the flow field will be also investigated in a Lagrangian framework. New experiments are planned using a wider range of variation of the experimental parameters and different types of forcing.

\section{Acknowledgements}

The authors would like to tank B. Galperin for useful discussions and suggestions. This work has been supported by the Italian MIUR-University Research Projects.

\section{References}

[1] G. K. Vallis and M. E. Maltrud, "Generation of mean flows and jets on a beta plane and over topography", J. Phys. Oceanogr., vol. 23, pp. 1346-1362, 1993.

[2] Y. D. Afanasyev and J. Wells, "Quasi-two-dimensional turbulence on the polar beta-plane: Laboratory experiments", Geophys. Astrophys. Fluid Dyn., vol. 99, pp.1-17, 2005.

[3] S. Espa, G. F. Carnevale, A. Cenedese and M. Mariani, "Quasi two-dimensional decaying turbulence subject to the $\beta$-effect”, J. Turbulence, vol. 9, pp. 1-18, 2008.

[4] S. Espa, G. Di Nitto and A. Cenedese, "The emergence of zonal jets in forced rotating shallow water turbulence: A laboratory study", Europh. Letters, vol. 92-34006, pp. 1-6, 2010 . 
[5] B. Galperin, S. Sukoriansky and H. P. Huang, "Universal n-5 spectrum of zonal flows on giant planets", Phys. Fluids, vol. 13 , pp. $1545-1548,2001$.

[6] H. Nakano, and H. Hasumi, "A series of jets embedded in the broad zonal flows in the Pacific obtained in eddy-permitting ocean general circulation models", J. Phys. Oceanogr., vol. 35, pp. 474-488, 2005.

[7] N. A. Maximenko, B. Bang, and H. Sasaki, "Observational evidence of alternating zonal jets in the world ocean", Geophys. Research Lett., vol. 32, L12607, 2005.

[8] P. B. Rhines, "Waves and turbulence on a beta plane", J. Fluid Mech., vol. 69, pp. 417-443, 2005.

[9] S. Sukoriansky, N. Dikovskaya and B. Galperin, "On the "arrest" of inverse energy cascade and the Rhines scale". J. Fluid Mech., vol. 64, pp. 3312-3327, 2007.

[10] S. Yoden and M. Yamada, "A numerical experiment on two-dimensional decaying turbulence on a rotating sphere", J. Atmos. Scie., vol. 50, pp. 631-643, 1993.

[11] J. Y. K. Cho and L. M. Polvani, "The emergence of jets and vortices in freely evolving, shallow-water turbulence on a sphere", Phys. Fluids, vol. 8(6), pp. 1531-1552, 1996.

[12] H.-P. Huang and W. A. Robinson, "Two-dimensional turbulence and persistent zonal jets in a global barotropic model", J. Atmos. Sciences, vol. 55, pp. 611-629, 1998.

[13] M. E. Maltrud and G. Vallis, "Energy spectra and coherent structures in forced two-dimensional and beta-plane turbulence”, J. Fluid. Mech., vol. 228, pp. 321-342, 1991.

[14] G. P. Williams, "Planetary circulations: 1. Barotropic representation of Jovian and terrestrial turbulence", J. Atmos. Scie., vol. 35, pp. 1399-1426, 1978.

[15] R. Scott and L. Polvani, "Forced-dissipative shallow-water turbulence on the sphere and the atmospheric circulation of the giant planets", J. Atmos. Sci., vol. 64, 3158-3176, 2007.

[16] T. Nozawa and S. Yoden, "Formation of zonal band structure in forced two-dimensional turbulence on a rotating sphere", Phys. Fluids, vol. 9, pp. 2081-2093, 1997.

[17] R. L. Panetta, "Zonal jets in wide baroclinically unstable regions: Persistence and scale selection", J. Atmos. Sci., vol. 50, pp. 2073-2106, 1993.

[18] B. Galperin, S. Sukoriansky, N. Dikovskaya, P. L. Read, Y. H. Yamazaki and R. Wordsworth, "Anisotropic turbulence and zonal jets in rotating flows with a $\beta$-effect", Nonlinear proc. in Geo., vol. 13, pp. 83-98, 2006.

[19] B. Galperin, S. Sukoriansky and N. Dikovskaya, "Zonostrophic turbulence", Phys. Scr. T132, 2008.

[20] S. Sukoriansky, B. Galperin and N. Dikovskaya, "Universal Spectrum of Two-Dimensional Turbulence on a Rotating Sphere and Some Basic Features of Atmospheric Circulation on Giant Planets". Phys. Rev. Lett., vol. 89, 12450, 2002.

[21] H. P. Huang, B. Galperin, S. Sukoriansky, "Anisotropic spectra in two-dimensional turbulence on the surface of a rotating sphere", Phys. of Fluids, vol. 13, pp. 225-240, 2001.

[22] B. Galperin, H. Nakano, H. P. Huang and S. Sukoriansky, "The ubiquitous zonal jets in the atmospheres of giant planets and Earth's oceans", Geophys. Res. Lett., vol. 31, L13303,
2004.

[23] A. Chekhlov, S. Orszag, S. Sukoriansky, B. Galperin and I. Staroselsky, "The effect of small-scale forcing on large-scale structures in two-dimensional flows". Physica D, vol. 98, pp. 321-334, 1996.

[24] J. A. Whitehead JR., "Mean flow generated by circulation on a $\beta$-plane: An analogy with the moving flame experiment", Tellus, vol. 27, pp. 358-364, 1975.

[25] R. Hide and P. J. Mason, "Sloping convection in a rotating fluid”, Adv. in Phys., vol. 24, pp. 47-100, 1975.

[26] M. E. Bastin and P. L. Read, "Experiments on the structure of baroclinic waves and zonal jets in an internally heated, rotating, cylinder of fluid", Phys. of Fluids, vol. 10, pp. 374-389, 1998.

[27] R. D. Wordsworth, P. L. Read and Y. H. Yamazaki, "Turbulence, waves, and jets in a differentially heated rotating anulus experiment", Phys. of fluids, vol. 20, 126602, 2008.

[28] J. Aubert, S. Jung, and H. L. Swinney, "Observations of zonal flow created by potential vorticity mixing in a rotating fluid", Geophys. Res. Lett., vol. 29(18), 1876, 2002.

[29] P. Tabeling, S. Burkhart, O. Cardoso and H. Willaime, "Experimental study of freely decaying two-dimensional turbulence”, Phys. Rev. Lett., vol. 67, pp. 3772-3775, 1991.

[30] H. J. H. Clercx, G. J. F. van Heijst and M. L. Zoeteweij, "Quasi-two-dimensional turbulence in shallow fluid layers: The role of bottom friction and fluid layer depth", Phys. Rev. E, vol. 67, 066303, 2003.

[31] G. Boffetta, A. Cenedese, S. Espa and S. Musacchio, "Effects of friction on 2D turbulence: an experimental study", Europhys. Letters, vol. 71(4), pp. 590-596, 2005.

[32] R. A. D. Akkermans, L. J. P. Kamp, H. J. H. Clercx and G. J. F. van Heijst, "Intrinsic three-dimensionality in electromagnetically driven shallow flows", Europhys. Lett., vol. 83, 24001, 2008.

[33] G. A. Voth, G. Haller, and J. P. Gollub, "Experimental Measurements of Stretching Fields in Fluid Mixing", Phys. Rev. Lett., vol. 88, 254501, 2002.

[34] N. F. Bondarenko, M. Z. Gak, and F. V. Dolzhanskiy, "Laboratory and theoretical models of plane periodic flows", Atmos. Oceanic Phys., vol. 15, pp. 711-716, 1979.

[35] S. Espa, A. Cenedese, M. Mariani and G. F. Carnevale, "Quasi-twodimensional flow on the polar $\beta$-plane: laboratory experiments”, J. Mar. Syst., vol. 77, pp. 502-510, 2009.

[36] S. Espa, I. Bordi, T. Frisius, K. Fraedrich, A. Cenedese and A. Sutera, "Zonal jets and cyclone/anticyclone asymmetry in decaying rotating turbulence: laboratory experiments and numerical simulations", Astrophys. Fluid Dyn., DOI:10.1080/03091929.2011.637301, 2012.

[37] P. L. Read, Y. H. Yamazaki, S. Lewis, P. Williams, R. Wordsworth, K. Miki-Yamazaki, J. Sommeria, H. Didelle and A. M. Fincham, "Dynamics of Convectively Driven Banded Jets in the Laboratory", J. Atmos. Sci., vol. 64, pp. 4031-4052, 2007.

[38] Y. D. Afanasyev, S. O'Leary, P. B. Rhines, and E. Lindahl, "On the origin of jets in the ocean", Geophys. and Astrophys. Fluid Dyn.,vol. 106(2), pp. 113-137, 2011. 
[39] A. G. Slavin and Y. D. Afanasyev, "Multiple zonal jets on the polar beta-plane", Phys. of Fluids, vol. 24, 016603, 2012 .

[40] J. Pedlosky, "Geophysical Fluid Dynamics". Springer-Verlag, New York Inc., 1987.

[41] M. Moroni and A. Cenedese, "Comparison among feature tracking and more consolidated velocimetry image analysis techniques in a fully developed turbulent channel flow", Meas. Sci. Technol., vol. 16, pp. 2307-2322, 2005.

[42] J. S. Bendat and A. G. Piersol, "Random Data-Analysis and Measurement Procedure" (ed. Barnett V. et al.), p. 106. Wiley and Sons, Inc, 2000. 